\title{
Detection of deltamethrin in cabbages using visible shortwave near-infrared spectroscopy
}

\author{
${ }^{1}$ Ishkandar, C.D.M., ${ }^{1,3, * N a w i, ~ N . M ., ~}{ }^{1}$ Janius, R., ${ }^{2}$ Mazlan, N., and ${ }^{4}$ Lin T.T. \\ ${ }^{1}$ Department of Biological and Agricultural Engineering, Faculty of Engineering, Universiti Putra \\ Malaysia, 43400 UPM Serdang, Selangor, Malaysia \\ ${ }^{2}$ Department of Agriculture Technology, Faculty of Agriculture, Universiti Putra Malaysia, 43400 UPM \\ Serdang, Selangor, Malaysia \\ ${ }^{3}$ Institute of Plantation Studies, Universiti Putra Malaysia, 43400 UPM Serdang, Selangor, Malaysia \\ ${ }^{4}$ Department of Bio-Industrial Mechatronics Engineering, National Taiwan University, Taipei 10617,
}

Taiwan

\begin{abstract}
Article history:
Received: 24 April 2020

Received in revised form: 15

December 2020

Accepted: 17 December 2020

Available Online: 13 June

2021
\end{abstract}

Keywords:

Cabbages,

Spectroscopy,

GC-ECD,

MRL,

Pesticide Residues

DOI:

https://doi.org/10.26656/fr.2017.5(3).742

\begin{abstract}
Pesticides have long been used in the cabbage industry to control pest infestation. This study investigated the potential application of low-cost and portable visible shortwave near-infrared spectroscopy for the detection of deltamethrin residue in cabbages. A total of sixty organic cabbage samples were used. The sample was divided into four batches, three batches were sprayed with deltamethrin pesticide whereas the remaining batch was not sprayed (control sample). The first three batches of the cabbages were sprayed with the pesticide at three different concentrations, namely low, medium and high with the values of $0.08,0.11$ and $0.14 \%$ volume/volume $(\mathrm{v} / \mathrm{v})$, respectively. Spectral data of the cabbage samples were collected using visible shortwave near-infrared (VSNIR) spectrometer with wavelengths range between 200 and $1100 \mathrm{~nm}$. Gas chromatography-electron capture detector (GC-ECD) was used to determine the concentration of deltamethrin residues in the cabbages. Partial least square (PLS) regression method was adopted to investigate the relationship between the spectral data and deltamethrin concentration values. The calibration model produced the values of coefficient of determination $\left(\mathrm{R}^{2}\right)$ and the root mean square error of calibration (RMSEC) of 0.98 and 0.02 , respectively. For the prediction model, the values of $\mathrm{R}^{2}$ and the root mean square error of prediction (RMSEP) were 0.94 and 0.04 , respectively. These results demonstrated that the proposed spectroscopic measurement is a promising technique for the detection of pesticide at different concentrations in cabbage samples.
\end{abstract}

\section{Introduction}

Vegetables are important food components for a healthy diet that can help in disease prevention. Cabbage (Brassicae oleracea var. capitata) is one of the most important and widely cultivated vegetables because of its nutritional and economical values. The world biggest cabbage producers were China, India and Russia with production amount around $32.8 \mathrm{Mt}, 8.2 \mathrm{Mt}$ and $3.3 \mathrm{Mt}$, respectively (FAOSTAT, 2017). In Malaysia, the production of cabbage in 2016 was $0.10 \mathrm{Mt}$ with a production value of about RM 180 million (Department of Agriculture, 2016).

In cabbage production, effective pest management is a very important factor to maximize farm productivity.
Typically, effective pest management is greatly associated with the use of chemical pesticide for controlling pests and diseases (Halimatunsadiah et al., 2016). Mazlan and Mumford (2005) reported that cabbage production was often affected by insects such as Myzus persicae, Plutella xylostella, and Phyllotreta striolata, which were responsible for causing crop loss up to $90 \%$.

Typically, farmers used pesticides to manage agricultural pests (Bommarco et al., 2011). Pesticides are chemical substances applied to crops at various stages of cultivation and during the post-harvest storage of crops (Bakırc1 et al., 2014). The use of pesticides is intended to prevent the destruction of food crops by controlling agricultural pests or unwanted plants and to improve 
plant quality (Bakırc1 and Hisil, 2012). However, it was reported that some farmers would overuse and apply pesticides rampantly to satisfy the high demand of the vegetable (Jipanin et al., 2001). As a result, serious problems were found to be increased including food poisoning, contaminated market produce and environmental pollution. In addition, incorrect application of pesticides may cause harm to the environment, increased resistance in the target pest organisms and deleterious effects on non-target organisms.

Typically, around $60 \%$ of farmers in Cameron Highlands used permethrin insecticide to control pests (Ismail and Kalithasan, 2004). A study by Jusoh et al. (1992) stated that $34.5 \%$ of cabbage samples in Malaysia exceeded the maximum residue limit (MRL) value. Another research conducted by Karadzic (2017) to detect deltamethrin pesticide in cabbages revealed that 0.01 to $0.20 \mathrm{mg} / \mathrm{kg}$ of MRL value were recorded, showing that a high concentration of pesticide contained in the samples. Other than that, a study by Zaidon et al. (2016) reported that MRL for cabbage samples at $0.00022 \mathrm{mg} / \mathrm{kg}$ may cause health problems if it was consumed in high quantity in the long terms.

To ensure the safety of the food and to protect consumer health, the monitoring of pesticide residues in food products must be pursued. Usually, the pesticide residues were measured in a laboratory using expensive technology such as gas chromatography with tandem mass spectrometry (GC-MS) (Osman et al., 2010; Palenikova et al., 2015) and liquid chromatography with tandem mass spectrometry (LC-MS) (Alez et al., 2008; Kaczyński and Lozowicka, 2015). According to Van Hoof et al. (2004), the multi-residue methods were used to detect the different analytes below or above the maximum residue limit (MRL). These measurement methods used to detect the presence of pesticide in agricultural product involves complex sample treatment since the vegetable need to be crushed and extracted to obtain an aliquot. Thus, a new non-destructive measurement method is required for rapid screening of pesticide presence in cabbage samples.

One of the most promising non-destructive techniques used to detect pesticide residue on agricultural produces is a spectroscopic method. For examples, this method was employed to detect pesticide residues in tomatoes (Saranwong and Kawano, 2005), peppers (Sanchez et al., 2010), navel oranges (Xue et al., 2012) and cucumbers (Jamshidi et al., 2016). This technique was non-contaminant, requiring low operating cost and having fast response time as compared to the conventional techniques (Montes et al., 2006).
Furthermore, it also requires little or no sample preparation (Peng et al., 2012). However, there is no published study regarding the application of the spectroscopic method to predict pesticide residues from cabbage samples. Thus, this study was conducted to investigate the potential application of visible shortwave near-infrared spectrometer to detect deltamethrin residues at different concentrations in cabbage samples.

\section{Materials and methods}

\subsection{Sample preparation}

A total of sixty organic cabbages were freshly purchased from a private farm located in Cameron Highlands, Malaysia. The cabbages which were harvested early in the morning were immediately delivered to the laboratory by using a refrigerator truck. The temperature of the refrigerator on the truck was set at $4^{\circ} \mathrm{C}$. On arrival at the laboratory, the cabbages were immediately stored in a chiller at the temperature of $3^{\circ} \mathrm{C}$ and $90 \%$ relative humidity. The average moisture content of the fresh cabbages was $92 \%$, similar to the value of fresh cabbages as reported by Velisek (2013).

Deltamethrin $\left(\mathrm{C}_{22} \mathrm{H}_{19} \mathrm{Br}_{2} \mathrm{NO}_{3}\right)$ which belongs to a pyrethroids pesticide group were used in this study. The pesticide was first diluted into three different concentrations indicated by volume per volume measurement (v/v) namely low $(0.08 \%(\mathrm{v} / \mathrm{v}))$, medium $(0.11 \%(\mathrm{v} / \mathrm{v}))$ and high $(0.14 \%(\mathrm{v} / \mathrm{v}))$. The cabbage samples were divided into four batches, where the first three batches were treated with the pesticide at low, medium and high concentrations. The fourth batch was not treated with any pesticide and regarded as a control sample. The pesticide was manually sprayed on the cabbage samples by using a knapsack sprayer with an average volume of $600 \mathrm{~mL}$ per sample. The nozzle sprayer was vertically positioned at approximately $15 \mathrm{~cm}$ from the samples. Pesticides were sprayed on the cabbages 24 hours before spectral scanning.

\subsection{Spectral acquisitions}

Before the spectral measurement, the cabbages were left on a bench until their temperature equilibrated with the room temperature at $25^{\circ} \mathrm{C}$. The reflectance spectra of each cabbage sample were recorded using a visible shortwave near-infrared (VSNIR) spectrometer (Ocean Optic HR4000, Ocean Optics Inc., Dunedin, Florida). This high-resolution miniature fibre optic spectrometer was equipped with the charge-coupled device (CCD) detector. The spectrometer was sensitive in the wavelength range between 200 and $1100 \mathrm{~nm}$ with an optical resolution of $0.025 \mathrm{~nm}$. The Spectrasuite software (Ocean Optic Inc., Dunedin, Florida) was installed on the 
computer for collecting, viewing and processing the spectra. The reflectance data were converted into absorbance data prior to data analysis. In order to avoid a low signal-to-noise ratio, some obvious noise was removed from both ends of the wavelength, considering only the wavelength regions between $500 \mathrm{~nm}$ and 980 $\mathrm{nm}$ for the calculations. Relative reflectance was calculated by dividing the reflectance of the cabbage samples with the reflectance from the white reference panel (WS-1 diffuse reflectance standard) for each wavelength.

\subsection{Sample scanning}

Each cabbage sample was placed inside a custommade measuring box for the spectral collection. The measuring box was used to enclose the sample and sensor from the ambient light which can affect the spectra (Lu et al., 2000). The inside surface of the box was painted black to minimize the influence of the background colour on the spectral data (Wu et al., 2008). The spectral measurement was conducted inside a black box which was illuminated by a halogen light source (Model: HL 2000, Ocean Optics Inc., Dunedin, Florida). After some trials, it was found that the best measurement distance between the probe and sample was $5 \mathrm{~mm}$. This scanning distance was fixed for every measurement. The cabbage samples were scanned at the most outer surface from different scanning locations (Figure 1). The spectral data from each scanning point was recorded three times and averaged before it was used in the development of calibration and prediction models.

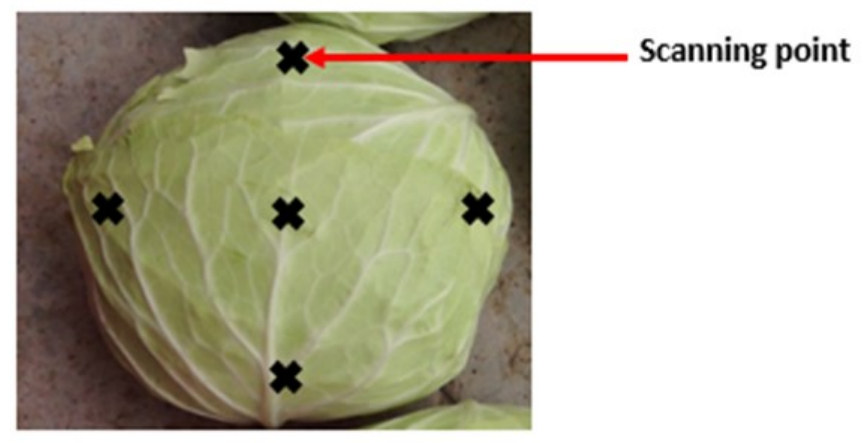

Figure 1. Scanning point on the top surface of the cabbage

\subsection{Gas chromatography}

After the spectral measurement, the samples were sent to the Department of Chemistry, Petaling Jaya, Malaysia for reference data analysis. The analysis was conducted using Agilent 7890A gas chromatograph (Agilent Technologies Inc., Santa Clara, California) equipped with an electron capture detector (GC-ECD) was used to determine the concentration level of deltamethrin in each cabbage sample. The extraction of synthetic pyrethroid followed the quick, easy, cheap, effective, rugged, and safe (QuEChERS) method
(Srivastava et al., 2014). The sample preparation and extraction for GC-ECD analysis were based on standard analysis procedures developed by Food and Agriculture Organization (2003) and Pakvilai et al. (2015).

\subsection{Pre-processing of spectral data}

The spectral data of solid samples is normally influenced by the skin roughness of the samples which can cause some problems in assessing their internal quality attributes (Lazim et al., 2016). Furthermore, instead of containing sample information, the spectral data may also contain background information such as random noise, light scattering, and path length variations. Raw spectral data may also comprise noise, uncertainties, variability, and un-recognized features (Moghimi et al., 2010). In order to obtain reliable, accurate and stable calibration models, it is essential to pre-process spectral data before modelling (Cen and He, 2007). There are several types of pre-processing methods were attempted in this study, including the smoothing technique of moving average, Savitzky-Golay, multiplicative scatter correlation (MSC), standard normal variation (SNV) transformation and normalization (Jayaselan et al., 2018). For comparison purposes, the raw spectral data without any pre-processing method was also analysed. The performance of the models developed using different pre-processing methods was compared with one another based on $\mathrm{R}^{2}$ and root means square error of predictions (RMSEP) values. All spectral data were pre-processed using Unscrambler $\mathrm{X}$ version 10.3 software (Camo Process, AS, Oslo, Norway).

\subsection{Development of calibration and validation models}

Prior to the development of calibration and validation models, principal component analysis (PCA) was applied in analyzing the spectra data to extract useful information, decrease the noise and reduce the number of principle components (PCs). PCA is a wellknown chemometrics technique to determine directions of maximum variability in sample grouping and uses them as new axes called principle components (PCs) that can be used as new variables, instead of the original data, in the following calculations (Blanco and Villarroya, 2002). PCA can also be used to detect spectral outliers that might affect model performance in each data set (Moghimi et al., 2010).

Partial least squares (PLS) analysis is commonly applied in near-infrared spectroscopy analysis. PLS analysis was employed to establish a regression model to predict concentration values of deltamethrin in the cabbage samples. The variable of matrix $\mathrm{X}$ (spectral data) and matrix $\mathrm{Y}$ (deltamethrin concentration) were simultaneously considered in the PLS analysis. In this 
study, PLS was applied as a regression method as well as a way to extract the latent variables (LVs). The LVs were considered as new eigenvectors of the original spectra to reduce the dimensionality and compress the original spectra data (Wu et al., 2008). A relatively low number of latent variables (LV) are desirable to avoid the modelling of signal noise (Gómez et al., 2006). The maximum numbers of LVs used in this study were set to ten.

Due to a limited number of cabbage samples, full cross-validation (leave-one-out) was applied to estimate the prediction quality and to avoid overfitting of the calibration model (Nawi et al., 2014). In this analysis, an external validation method was also applied to examine the performance of the PLS models. The external validation procedure determines the predictive ability of an equation from the sample sets which were not used in the calibration model. Before the calibration, the samples were divided into two sets; $75 \%$ of the samples were used to develop a prediction equation while the remaining $25 \%$ were used to validate the prediction equation. Samples for validation were selected by taking one of every four samples from the entire sample set, taking care to ensure that each set included samples that covered the whole range of the deltamethrin concentration values of the cabbages (Nawi et al., 2013).

In this paper, both PCA for PLS modelling were run using the Unscrambler $\mathrm{X}$ version 10.3 software (Camo Process, AS, Oslo, Norway). The performance of the final PLS models was evaluated by the coefficient of determination for calibration $\left(\mathrm{R}^{2}\right)$, root mean squares of calibration (RMSEC), and the coefficient of determination for prediction $\left(\mathrm{R}^{2}\right)$ and root mean squares of prediction (RMSEP). A proper model should have a low RMSEC, RMSEP and a high $\mathrm{R}^{2}$ for both calibration and prediction models (Abdullah et al., 2019).

\section{Results and discussion}

\subsection{Samples properties and the spectral characteristics}

The summary of the statistical values of deltamethrin concentration for the calibration and prediction data sets is shown in Table 1. T-test results showed that the prediction mean values and calibration mean values of deltamethrin residues obtained from GC-ECD were significantly different with $p$-value $=0.007(p<0.05)$. A relatively wide range of concentration values obtained from standard deviation (SD) for the cabbage samples was due to the different concentrations of the deltamethrin sprayed on the cabbages. However, the mean values of the concentration were approximately similar for the calibration and prediction data sets.

Table 2 shows the values of deltamethrin
Table 1. Statistics of deltamethrin concentration $(\mathrm{mg} / \mathrm{kg})$ found in the cabbage samples

\begin{tabular}{lccccc}
\hline Sample sets & $\mathrm{n}$ & Minimum & Maximum & Mean & $\mathrm{SD}$ \\
\hline Calibration & 45 & 0.018 & 0.846 & 0.219 & 0.223 \\
Prediction & 15 & 0.024 & 0.890 & 0.206 & 0.255 \\
Total sample & 60 & 0.018 & 0.890 & 0.216 & 0.229 \\
\hline
\end{tabular}

$\mathrm{n}=$ number of sample, $\mathrm{SD}=$ standard deviation

Table 2. Residue concentration detected by GC-ECD at different treatment categories

\begin{tabular}{cc}
\hline Treatment Category & Residue concentration $(\mathrm{mg} / \mathrm{kg})$ \\
\hline Low & 0.06 \\
Medium & 0.17 \\
High & 0.41 \\
No pesticide & 0.00 \\
\hline
\end{tabular}

concentration obtained from the GC-ECD analysis. A low concentration of the pesticide $(0.08 \% \mathrm{v} / \mathrm{v})$ sprayed on the cabbages produced low concentration of deltamethrin value $(0.06 \mathrm{mg} / \mathrm{kg})$. The same trend can be observed for the deltamethrin at medium and high concentrations. According to the Department of Agriculture Malaysia (2015), the MRL permitted for deltamethrin residue in food is $0.2 \mathrm{mg} / \mathrm{kg}$. For this table, it can be concluded that the cabbages sprayed with a high concentration of deltamethrin had the highest value of residue concentration at $0.41 \mathrm{mg} / \mathrm{kg}$, exceeding the safe limit of MRL set by the government.

\subsection{Typical absorbance spectra of cabbages at different pesticides concentration}

Figure 2 shows typical absorbance spectra of the cabbage samples without pesticide treatment $(0.00 \mathrm{mg} /$ $\mathrm{kg}$ ) and treated with deltamethrin pesticide at different concentration levels namely low $(0.06 \mathrm{mg} / \mathrm{kg})$, medium $(0.17 \mathrm{mg} / \mathrm{kg})$ and high $(0.41 \mathrm{mg} / \mathrm{kg})$. It can be seen that the trend of the curve is similar for all treatments. However, the cabbage samples treated with a high pesticide concentration has a relatively lower absorbance value. The difference in the absorbance values was due to the different chemical composition in each pesticide (Zacharia, 2011). Jamshidi et al. (2016) reported that different pesticide concentration could change the value

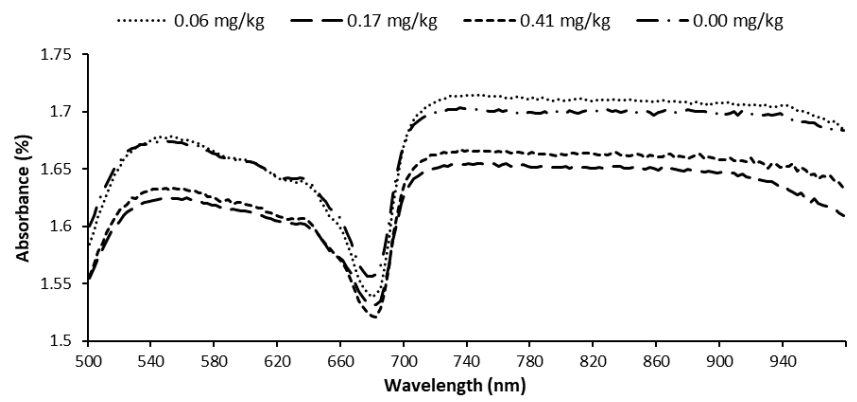

Figure 2. Typical absorbance spectra of the cabbage samples with and without deltamethrin treatment 
of the spectral data. It can also be seen that the spectral curve steadily increases from 680 to $735 \mathrm{~nm}$ since the samples absorbed red light in the visible range (Coops et al., 2003; Datt, 1999). The near-infrared (NIR) region from 750 to $940 \mathrm{~nm}$ also shows that a high concentration of pesticide has a low absorbance value.

The difference between the spectra curves was obvious in the visible region $(500-750 \mathrm{~nm})$. In this region, the absorbance values decreased along with the wavelength until they reached the lowest point around $682 \mathrm{~nm}$. The spectral curve at $682 \mathrm{~nm}$ can be related to the chlorophyll content of the cabbages. This spectral curve might be used as an effective wavelength as it shows the difference of absorbance values corresponding to different pesticide concentration levels. $\mathrm{Li}$ and $\mathrm{He}$ (2008) reported that the wavelength regions around 435, $450,488,523,550,615,650,675,695,931,970$, and $989 \mathrm{~nm}$ could be used to determine the chlorophyll concentration.

\subsection{Effects of different preprocessing methods on prediction accuracy}

The spectral pre-processing technique is an important step to remove any irrelevant information including noise, uncertainties, variability, interactions and unrecognized features (Lazim et al., 2016). The influence of selected different spectra pre-processing methods on the PLS model performance for residue detection in the cabbage samples is shown in Table 3. For comparison purposes, the PLS model performance for spectral data without any pre-processing method (raw spectral data) is also shown. From the table, it can be seen that if no pre-processing method was applied, the $\mathrm{R}^{2}$ values for calibration and prediction models were 0.97 and 0.93 , respectively. With the pre-processing methods, higher $\mathrm{R}^{2}$ values for the prediction models were obtained by using smoothing (Gaussian Filter) $\left(\mathrm{R}^{2}=0.97\right)$, smoothing (Savitzky-Golay) $\left(\mathrm{R}^{2}=0.95\right)$ and standard normal variate $\left(\mathrm{R}^{2}=0.97\right)$. Since the RMSEP value for smoothing (Savitzky-Golay) was the lowest (0.04), the Table 3. The influence of different pre-processing methods on the performance of the PLS models

\begin{tabular}{lccccc}
\hline \multicolumn{1}{c}{ Pre-processing } & \multirow{2}{*}{$\mathrm{LV}$} & \multicolumn{2}{c}{ Calibration } & \multicolumn{2}{c}{ Prediction } \\
& & $\mathrm{R}^{2}$ & $\mathrm{RMSEC}$ & $\mathrm{R}^{2}$ & $\mathrm{RMSEP}$ \\
\hline $\begin{array}{l}\text { Raw data (without } \\
\text { pre-processing) }\end{array}$ & 10 & 0.97 & 0.03 & 0.93 & 0.04 \\
$\begin{array}{l}\text { Smoothing } \\
\text { (Gaussian Filter) }\end{array}$ & 10 & 0.93 & 5.92 & 0.97 & 4.73 \\
$\begin{array}{l}\text { Smoothing (Savitzky } \\
\text {-Golay) }\end{array}$ & 10 & 0.98 & 0.02 & 0.95 & 0.04 \\
$\begin{array}{l}\text { Standard Normal } \\
\text { Variate (SNV) }\end{array}$ & 10 & 0.97 & 0.06 & 0.97 & 0.06 \\
\hline
\end{tabular}

$\mathrm{LV}=$ latent variable; $\mathrm{RMSEC}=$ root mean square error calibration; $\mathrm{RMSEP}=$ root mean square error prediction; $\mathrm{R} 2=$ correlation of determination output from this method was chosen for further discussion in this paper. Smoothing (Savitzky-Golay) performed well in this study because this method is commonly applied to eliminate the slope of the spectral data (Swierenga et al., 1999).

\subsection{Pattern recognition of the deltamethrin residues on cabbages}

Figure 3 shows the result of pattern recognition developed using the PCA classification method for deltamethrin pesticide at different concentrations. PCA can be applied to display the hidden structure and gives insights into how measured variables cause some similarity or differences among samples from each other (Li et al., 2007). The spectral data used to develop this classification was pre-treated using the Savitzky-Golay smoothing method. The figure shows that a clear separation between deltamethrin at different concentrations, with the most obvious one, belongs to deltamethrin at the highest concentration (DH). However, some overlapping values can be seen among NP, DL, and DM. This figure can be used to examine the qualitative differences of deltamethrin at different concentrations.

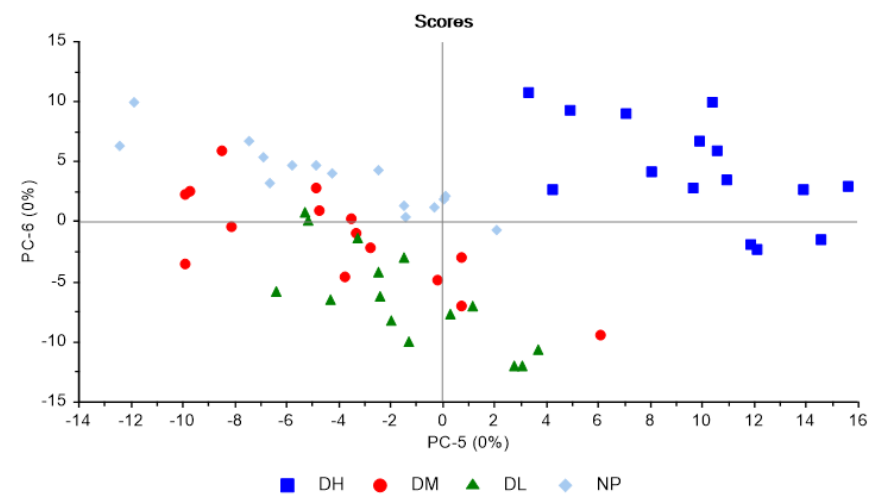

Figure 3. PCA classification of deltamethrin pesticide at different concentrations

\subsection{The prediction of MRL from cabbage samples}

PLS models were developed using the pre-processed absorbance spectra by smoothing (Savitzky-Golay) technique. The value of these indices for both calibration and prediction models is shown in the scatter plot in Figure 4. In the figure, the ordinate and abscissa represent the predicted and measured concentration values of deltamethrin. For the calibration model, the $\mathrm{R}^{2}$ and RMSEC values obtained were 0.98 and 0.02 , respectively. For the prediction model, the excellent values for $\mathrm{R}^{2}$ and RMSEP were obtained, with the values being 0.95 and 0.04 , respectively. Shenk and Westerhaus (1996) suggested that the $R^{2}$ value greater than 0.9 indicates excellent quantitative information of the models. 


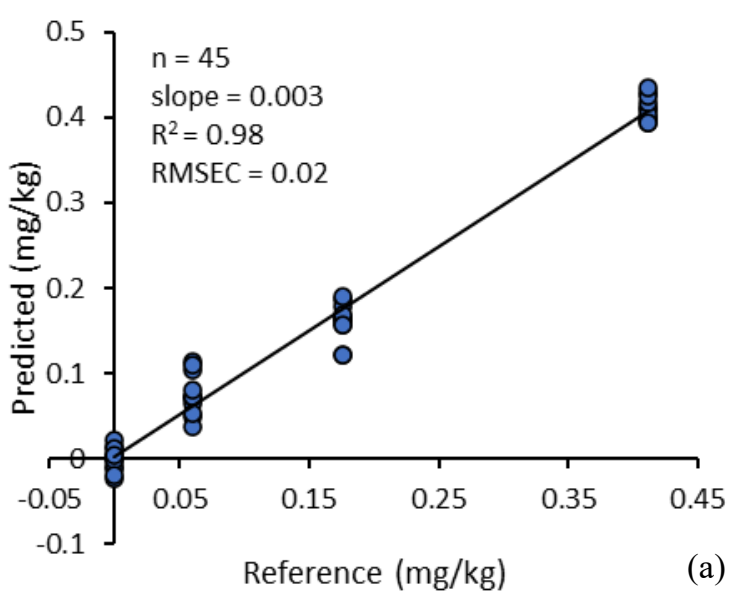

(a)

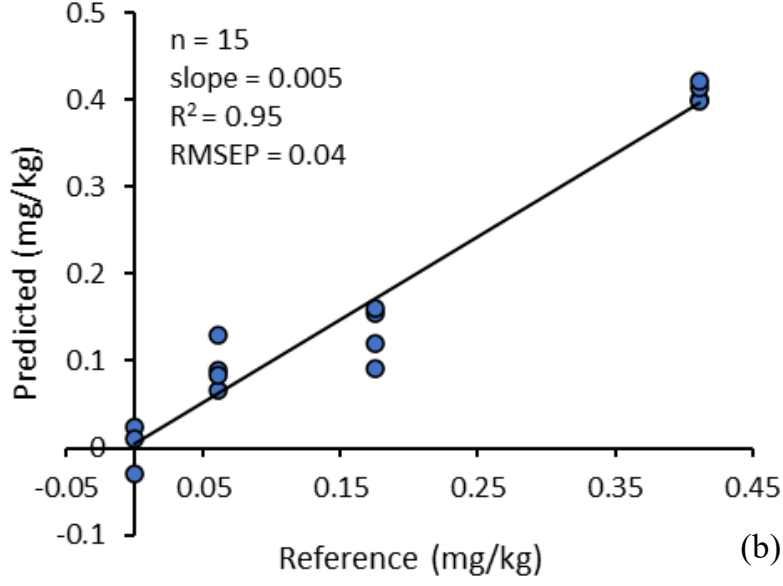

(b)

Figure 4. Prediction versus reference values of deltamethrin concentration for a) calibration model; b) prediction model.

In addition, the prediction value obtained in this study was better than the results of the similar study reported by Xue et al. (2012) who predicted dichlorvos pesticide residue in navel orange. In their research, the authors reported that the prediction model with the $\mathrm{R}^{2}$ value of 0.87 . A similar study was also conducted by Jamshidi et al. (2016) who employed VIS-NIR spectroscopy to predict pesticide from cucumbers. The authors reported the $\mathrm{R}^{2}$ value for the prediction model in their study was 0.91 with a standard error of crossvalidation (SECV) of 3.22. By comparing the PLS results from previous researches, this study has shown considerably good potential for predicting deltamethrin in cabbage samples.

\section{Conclusion}

This study has shown that the VSNIR spectrometer has the potential to detect the presence of deltamethrin at different concentrations in the cabbage samples. Some pre-processing methods could improve the prediction capability of the models. The correlation between the spectral data and deltamethrin concentrations yielded excellent results for both calibration and prediction models with $\mathrm{R}^{2}$ values of 0.98 and 0.94 , respectively. However, the results in this study were obtained based on limited cabbage samples collected from only one harvest season. The samples also subjected to the same agronomy practices under the same weather conditions. Thus, it is recommended that this study be repeated and replicated using more cabbage samples collected from different farms with different agronomic practices over several harvest seasons.

\section{Acknowledgements}

The authors acknowledge the Ministry of Higher Education, Malaysia for providing research grant under FRGS (FRGS/1/2015/TK04/UPM/02/6) and scholarships under the MyBrain Program. The authors also acknowledge Universiti Putra Malaysia for providing the
Graduate Research Fellow scheme to support this research.

\section{References}

Abdullah, N., Nawi, N.M.,Ding, P., Kassim. M.S.M. and Lazim, S.S.R.M (2019). Preliminary study to predict moisture content of jackfruit skin using shortwave near-infrared spectroscopy. Food Research, 3(2), 128 - 132. https://doi.org/10.26656/fr.2017.3(2).135

Bakırcı, G.T. and Hış1, Y. (2012). Fast and simple extraction of pesticide residues in selected fruits and vegetables using tetrafluoroethane and toluene followed by ultrahigh-performance liquid chromatography/tandem mass spectrometry. Food Chemistry, 135(3), 1901-1913. https:// doi.org/10.1016/j.foodchem.2012.06.051

Bakırcı, G.T., Acay, D.B.Y., Bakırcı, F. and Ötleş, S. (2014). Pesticide residues in fruits and vegetables from the Aegean region, Turkey. Food Chemistry, 160, 379-392. https://doi.org/10.1016/ j.foodchem.2014.02.051

Blanco, M. and Villarroya, I. (2002). NIR Spectroscopy: A Rapid-Response Analytical Tool. Trends in Analytical Chemistry, 21(4), 240-250. https:// doi.org/10.1016/S0165-9936(02)00404-1

Bommarco, R., Miranda, F., Bylund, H. and Björkman, C. (2011). Insecticides suppress natural enemies and increase pest damage in cabbage. Journal of Economic Entomology, 104(3), 782-791. https:// doi.org/10.1603/EC10444

Cen, H. and He, Y. (2007). Theory and Application of Near-infrared Reflectance Spectroscopy in Determination of Food Quality. Trends in Food Science and Technology, 18(2), 72-83. https:// doi.org/10.1016/j.tifs.2006.09.003

Coops, N.C., Stone, C., Culvenor, D.S., Chisholm, L.A. and Merton, R.N. (2003). Chlorophyll Content in Eucalypt Vegetation at the Leaf and Canopy Scales as Derived from High Resolution Spectral Data. Tree 
Physiology, 23(1), 23-31. https://doi.org/10.1093/ treephys/23.1.23

Datt, B. (1999). A new reflectance index for remote sensing of chlorophyll content in higher plants: tests using Eucalyptus leaves. Journal of Plant Physiology, 154(1), 30-36. https://doi.org/10.1016/ S0176-1617(99)80314-9

Department of Agriculture. (2015). Summary book of pesticide residue for food crops (Akta Makanan 1983). Putrajaya, Malaysia: Department of Agriculture.

Department of Agriculture. (2016). Vegetables and Cash Crops Statistics Malaysia. Retrieved from http:// www.doa.gov.my/c/document_library/get_file?uuid $=$ 111 ca31244ff\&groupId $=38257$

816658e9-f178-4d16-a896-

FAOSTAT (2017). Agriculture Data. Retrieved on June 2, 2017 from FAOSTAT website: http:// faostat.fao.org.

Food and Agriculture Organization. (2003). Guidelines on Good Laboratory Practice in Residue Analysis. Food and Agriculture Organization and World Health Organization CAC/GL 40-1993.

Gómez, A.H., He, Y. and Pereira, A.G. (2006). Nondestructive measurement of acidity, soluble solids and firmness of Satsuma mandarin using Vis/NIRspectroscopy techniques. Journal of food engineering, 77(2), 313-319. https://doi.org/10.1016/ j.jfoodeng.2005.06.036

Halimatunsadiah, A.B., Norida, M., Omar, D. and Kamarulzaman, N.H. (2016). Application of pesticide in pest management: The case of lowland vegetable growers. International Food Research Journal, 23(1), 85-94.

Ismail, B.S. and Kalithasan, K. (2004). Adsorption, Desorption and Mobility of Permethrin in Malaysian Soils. Journal of Environmental Science and Health, Part B, 39(3), 419-429. https://doi.org/10.1081/PFC120035927

Jamshidi, B., Mohajerani, E. and Jamshidi, J. (2016). Developing a Vis/NIR Spectroscopic System for Fast and Non-Destructive Pesticide Residue Monitoring in Agricultural Product. Measurement, 89, 1-6. https:// doi.org/10.1016/j.measurement.2016.03.069

Jayaselan, H.A.J., Ismail, W.I.W., Nawi, N.M. and Shariff, A.R.M. (2018). Determination of the Optimal Pre-processing Technique for Spectral Data of Oil Palm Leaves with Respect to Nutrient. Pertanika Journal of Science and Technology, 26(3), 1169-1182.

Jipanin, J., Rahman, A.A., Jaimi, J.R. and Phua, P.K.
(2001). Management of Pesticide Use on Vegetable Production: Role of Department of Agriculture Sabah. Presented at the 6th SITE Research Seminar, 13 - 14 September 2001.

Jusoh, M.M. (1992). Training Manual on Integrated Pest Management of Diamondback Moth in Cabbage in Malaysia. Malaysia: Malaysian Agricultural Research and Development Institute.

Kaczyński, P. and Łozowicka, B. (2015). Liquid chromatographic determination of glyphosate and aminomethylphosphonic acid residues in rapeseed with MS/MS detection or derivatization/fluorescence detection. Open Chemistry, 13, 1011-1019. https:// doi.org/10.1515/chem-2015-0107

Karadzic M. (2017). Pesticide Residues in Cabbage. Review of Scientific Paper of the Student of Agronomy. Vol. 10, p. 484-493. Cacak, Serbia: Faculty of Agronomy, University of Kragujevac.

Lazim, S.S.R.M., Nawi, N.M., Chen, G., Jensen, T. and Rasli, A.M.M. (2016). Influence of different preprocessing methods in predicting sugarcane quality from near-infrared (NIR) spectral data. International Food Research Journal, 23, S231.

Li, X., He, Y. and Fang, H. (2007). Non-destructive discrimination of Chinese bayberry varieties using Vis/NIR spectroscopy. Journal of Food Engineering, 81(2), 357-363. https://doi.org/10.1016/ j.jfoodeng.2006.10.033

Lu, R., Guyer, D. and Beaudry, R.M. (2000). Determination of firmness and sugar content of apple using NIR diffuse absorbance. Journal of Texture Studies, 31(6), 615-630. https://doi.org/10.1111/ j.1745-4603.2000.tb01024.x

Mazlan, N. and Mumford, J. (2005). Insecticide use in cabbage pest management in the Cameron Highlands, Malaysia. Crop Protection, 24(1), 31-39. https://doi.org/10.1016/j.cropro.2004.06.005

Moghimi, A., Aghkhani, M.H., Sazgarnia, A. and Sarmad, M. (2010). Vis/NIR spectroscopy and chemometrics for the prediction of soluble solids content and acidity $(\mathrm{pH})$ of kiwifruit. Biosystems Engineering, 106(3), 295-302. https:// doi.org/10.1016/j.biosystemseng.2010.04.002

Montes, J.M., Utz, H.F., Schipprack, W., Kusterer, B., Muminovic, J., Paul, C. and Melchinger, A.E. (2006), 'Near-infrared spectroscopy on combine harvesters to measure maize grain dry matter content and quality parameters, Plant Breeding 125(6), 591595. https://doi.org/10.1111/j.14390523.2006.01298.x

Nawi, N.M., Chen, G. and Jensen, T. (2014). Prediction of sugarcane quality from juice samples using 
portable spectroscopy. Journal of Mechanical Engineering and Sciences, 7, 1219-1226. https:// doi.org/10.15282/jmes.7.2014.21.0119

Nawi, N.M., Chen, G., Jensen, T. and Mehdizadeh, S.A. (2013). Prediction and classification of sugar content of sugarcane based on skin scanning using visible and shortwave near-infrared. Biosystems Engineering, 115(2), 154-161. https:// doi.org/10.1016/j.biosystemseng.2013.03.005

Osman, K.A., Al-Humaid, A.M., Al-Rehiayani, S.M. and Al-Redhaiman, K.N. (2010). Monitoring of pesticide residues in vegetables marketed in Al-Qassim region, Saudi Arabia. Ecotoxicology and Environmental Safety, 73(6), 1433-1439. https:// doi.org/10.1016/j.ecoenv.2010.05.020

Pakvilai, N., Prapamontol, T., Thavornyutikarn, P., Mangklabruks, A., Chantara, S., Hongsibsong, S. and Santasup, C. (2015). A Simple and Sensitive GC -ECD Method for Detecting Synthetic Pyrethroid Insecticide Residues in Vegetable and Fruit Samples. Chiang Mai Journal of Science, 42(1), 196 $-207$.

Peng, Y., Li, Y. and Chen, J. (2012.). Optical Technologies for Determination of Pesticide Residue. In Theophile, T. (Ed.) Infrared Spectroscopy - Materials Science, Engineering and Technology. InTech Open E-Book. https:// doi.org/10.5772/37575

Sánchez, M.T., Flores-Rojas, K., Guerrero, J.E., GarridoVaro, A. and Pérez-Marín, D. (2010). Measurement of pesticide residues in peppers by near-infrared reflectance spectroscopy. Pest Management Science: formerly Pesticide Science, 66(6), 580-586. https:// doi.org/10.1002/ps.1910

Saranwong, I. and Kawano, S. (2005). Rapid Determination of Fungicide Contaminated on Tomato Surfaces using the DESIR-NIR: A System for Ppm-Order Concentration. Journal of Nearinfrared Spectroscopy, 13(3), 169-175. https:// doi.org/10.1255/jnirs.470

Shenk, J.S. and Westerhaus, M.O. (1996). Calibration the ISI way. In Davis, A.M.C. and Williams, P. (Eds.) Near-infrared spectroscopy: The future waves, p. 198-202. Chichester, England, United Kingdom: NIR Publications.

Swierenga, H., De Weijer, A.P., Van Wijk, R.J. and Buydens, L.M.C. (1999). Strategy for constructing robust multivariate calibration models. Chemometrics and Intelligent Laboratory Systems, 49(1), 1-17. https://doi.org/10.1016/S01697439(99)00028-3

Van Hoof, N., De Wasch, K., Poelmans, S., Noppe, H. and De Brabander, H. (2004). Multi-residue liquid chromatography/tandem mass spectrometry method for the detection of non-steroidal anti-inflammatory drugs in bovine muscle: Optimisation of ion trap parameters. Rapid Communications in Mass Spectrometry, 18(23), 2823-2829. https:// doi.org/10.1002/rcm.1683

Velisek, J. (2013). The Chemistry of Food. United Kingdom: John Wiley \& Sons.

Wu, D., Feng, L., Zhang, C. and He, Y. (2008). Early Detection of Botrytis Cinerea on Eggplant Leaves based on Visible and Near-Infrared Spectroscopy. Transactions of the ASABE, 51(3), 1133-1139. https://doi.org/10.13031/2013.24504

Xue, L., Cai, J., Li, J. and Liu, M. (2012). Application of Particle Swarm Optimization (PSO) Algorithm to Determine Dichlorvos Residue on the Surface of Navel Orange with Vis-NIR Spectroscopy. Procedia Engineering, 29, 4124-4128. https://doi.org/10.1016/ j.proeng.2012.01.631

Zacharia, J.T. (2011). Identity, Physical and Chemical Properties of Pesticides. In Stoytcheva, M. (Ed.) Pesticides in the Modern World-Trends in Pesticides Analysis. InTech Open E-Book. https:// doi.org/10.5772/17513

Zaidon, S.Z., Hamsan, H and Bin, Y. (2016). A Review on Pesticides Occurrence in Fruits and Vegetables in Malaysia and Their Potential Health Risk among Adults. Indian Journal of Environmental Protection, 36(10), 826-832. 